\title{
Gut Microbiome Alterations Precede Cerebral Amyloidosis and Microglial Pathology in a Mouse Model of Alzheimer's Disease
}

\author{
Yijing Chen $\mathbb{D}^{1},{ }^{1}$ Lihua Fang, ${ }^{2}$ Shuo Chen, ${ }^{2}$ Haokui Zhou, ${ }^{2}$ Yingying Fan, ${ }^{1}$ Li Lin, ${ }^{1}$ Jing Li, ${ }^{1}$ \\ Jinying $\mathrm{Xu},{ }^{1,3}$ Yuewen Chen, ${ }^{1,3,4}$ Yingfei Ma $\mathbb{D}^{2},{ }^{2}$ and Yu Chen $\mathbb{D D}^{1,3,4}$ \\ ${ }^{1}$ The Brain Cognition and Brain Disease Institute, Shenzhen Institutes of Advanced Technology, Chinese Academy of Sciences, \\ Shenzhen-Hong Kong Institute of Brain Science-Shenzhen Fundamental Research Institutions, Shenzhen, Guangdong, China \\ ${ }^{2}$ Institute of Synthetic Biology, Shenzhen Institutes of Advanced Technology, Chinese Academy of Sciences, Shenzhen, \\ Guangdong, China \\ ${ }^{3}$ University of Chinese Academy of Sciences, Beijing, China \\ ${ }^{4}$ Guangdong Provincial Key Laboratory of Brain Science, Disease and Drug Development, HKUST Shenzhen Research Institute, \\ Shenzhen-Hong Kong Institute of Brain Science-Shenzhen Fundamental Research Institutions, Shenzhen, Guangdong, China
}

Correspondence should be addressed to Yingfei Ma; yingfei.ma@siat.ac.cn and Yu Chen; yu.chen@siat.ac.cn

Received 10 February 2020; Revised 11 April 2020; Accepted 15 April 2020; Published 27 May 2020

Academic Editor: Bence Racz

Copyright (c) 2020 Yijing Chen et al. This is an open access article distributed under the Creative Commons Attribution License, which permits unrestricted use, distribution, and reproduction in any medium, provided the original work is properly cited.

\begin{abstract}
Emerging evidence suggests that the gut microbiome actively regulates cognitive functions and that gut microbiome imbalance is associated with Alzheimer's disease (AD), the most prevalent neurodegenerative disorder. However, the changes in gut microbiome composition in $\mathrm{AD}$ and their association with disease pathology, especially in the early stages, are unclear. Here, we compared the profiles of gut microbiota between APP/PS1 transgenic mice (an AD mouse model) and their wild-type littermates at different ages by amplicon-based sequencing of $16 \mathrm{~S}$ ribosomal RNA genes. Microbiota composition started diverging between the APP/PS1 and wild-type mice at young ages (i.e., 1-3 months), before obvious amyloid deposition and plaque-localized microglial activation in the cerebral cortex in APP/PS1 mice. At later ages (i.e., 6 and 9 months), there were distinct changes in the abundance of inflammationrelated bacterial taxa including Escherichia-Shigella, Desulfovibrio, Akkermansia, and Blautia in APP/PS1 mice. These findings suggest that gut microbiota alterations precede the development of key pathological features of $\mathrm{AD}$, including amyloidosis and plaque-localized neuroinflammation. Thus, the investigation of gut microbiota might provide new avenues for developing diagnostic biomarkers and therapeutic targets for $\mathrm{AD}$.
\end{abstract}

\section{Introduction}

Alzheimer's disease (AD) is a neurodegenerative disease and form of dementia that severely impairs cognitive functions and daily activities. In $\mathrm{AD}$, the major pathological changes in the brain are elevated levels of extracellular amyloid plaques and intracellular neurofibrillary tangles [1]. The combinatorial effects of genetic and environmental factors are thought to contribute to the disease pathogenesis. Moreover, recent accumulating evidence suggests that gut microbiota dysbiosis and microbial infection might be associated with $\mathrm{AD}$ etiology [2-4].
The complex gut microbiota in the mammalian gastrointestinal ecosystem participates in a plethora of physiological processes $[5,6]$. Pathological changes in the gut microbiome not only lead to gut dysfunction but also are associated with central nervous system (CNS) disorders such as neurodegeneration, autism, and depression [7-11]. Gut microbiota can affect CNS functions through multiple ways, for example, by releasing neurotransmitters (e.g., acetylcholine, GABA, dopamine, and serotonin) and endotoxins that gain access to the brain via blood circulation, triggering the secretion of proinflammatory cytokines (e.g., IL-1 $\beta$, IL-6, and IL-17A) or anti-inflammatory cytokines (e.g., IL-4 and IL-10) by 
TABLE 1: Number of mouse fecal samples collected in each age group.

\begin{tabular}{lccccc}
\hline Mouse group & 1 month $(n)$ & 2 months $(n)$ & 3 months $(n)$ & 6 months $(n)$ & 9 months $(n)$ \\
\hline WT mice & 14 & 17 & 17 & 31 & 34 \\
APP/PS1 mice & 21 & 24 & 24 & 18 & 18 \\
\hline
\end{tabular}

WT: wild type.

mucosal immune cells that regulate immune activity in the CNS, as well as activating the gut vagus nerve, which influences multiple brain regions involving the limbic cortex, hippocampus, amygdala, striatum, and hypothalamus [12-15]. In turn, the CNS might influence gut microbiota composition through the reciprocal gut-brain axis: stress and negative emotions such as anxiety and depression might cause the release of neurotransmitters (including noradrenaline, adrenaline, and corticosterone) through the hypothalamicpituitary-adrenal axis and sympathetic nervous system, which could influence gut physiology and alter microbiota composition [16, 17]. In contrast, positive emotions such as happiness and positive affect are associated with lower expression of inflammatory genes in the immune cells and lower levels of proinflammatory cytokines such as IL-6 and TNF- $\alpha$ [18-20].

Emerging evidence suggests that microbial infection is a key factor in the etiopathogenesis of $\mathrm{AD}$, opening new avenues for therapeutic development [21]. Indeed, recent studies revealed that gut microbiota composition and diversity are altered in $\mathrm{AD}$ patients and animal models [2, 22-27]. Moreover, pathogenic microbes can induce amyloid-beta $(\mathrm{A} \beta)$ oligomerization and promote chronic inflammatory responses that contribute to $\mathrm{AD}$ progression $[4,28,29]$. However, there is a lack of evidence that the microbiota is altered in early $\mathrm{AD}$ or in the long term. Therefore, the changes in the microbiota profiles in $\mathrm{AD}$ require further investigation.

In the present study, we investigated longitudinal changes in the gut microbiome in an AD mouse model starting from an early age. We found that compared to wild-type littermates, the microbiota composition in $\mathrm{AD}$ mice began to diverge significantly from an early age (before the detection of $A \beta$ plaques in the cortex) and subsequently diverged even further. Thus, our findings suggest that gut microbiota composition may be associated with the progression of $\mathrm{AD}$ pathology.

\section{Materials and Methods}

2.1. Animals and Sample Collection. We obtained APP/PS1 double-transgenic mice (B6C3-Tg(APPswe, PSEN1dE9)85Dbo/J; stock number 2010-0001), a mouse model of $\mathrm{AD}$ in a C57BL/6 background, from the Nanjing Biomedical Research Institute of Nanjing University. We housed APP/PS1 mice and their age-matched wild-type (WT) littermates together ( $n=4$ mice/cage) under specific pathogen-free conditions and at a constant temperature $\left(24^{\circ} \mathrm{C}\right)$ in a $12 \mathrm{~h} \mathrm{light} /$ dark cycle, with autoclaved water and standard chow ad libitum. We collected fresh fecal samples from individual male mice at 1,2,3, 6 , and 9 months of age $(n=14-24$ for the 1-, 2-, 3-, and 9-month-old groups, $n=31-34$ for the 6-month-old group; Table 1), froze them immediately, and stored them at $-80^{\circ} \mathrm{C}$ before analysis. This study was performed in accordance with the recommendations of the National Care and Use of Animals Guidelines (China) and approved by the Institutional Animal Care and Use Committee (IACUC) of the Shenzhen Institutes of Advanced Technology, Chinese Academy of Sciences.

2.2. Fecal DNA Extraction and Sequencing. We extracted fecal DNA from mouse feces using the DNeasy PowerSoil Kit (QIAGEN, USA) according to the manufacturer's instructions. We measured the concentration and quality of the extracted DNA by NanoDrop 2000 (NanoDrop Technologies, Thermo Scientific, USA) and $1.2 \%$ agarose gel electrophoresis. We subsequently amplified $16 \mathrm{~S}$ ribosomal RNA (16S rRNA) genes with a set of primers that target the V3-V4 hypervariable regions of the bacterial 16S rDNA (see Supplementary Table S1 for detailed information). We performed polymerase chain reaction (PCR) with PCR mix (Transgene, China), fecal DNA template, primers, and sterilized ultrapure water in a total volume of $50 \mu \mathrm{L}$ according to the manufacturer's instructions. The cycling conditions were as follows: initial denaturation at $95^{\circ} \mathrm{C}$ for 5 minutes; 35 cycles of denaturation at $95^{\circ} \mathrm{C}$ for 30 seconds, annealing at $60^{\circ} \mathrm{C}$ for 30 seconds, and extension at $72^{\circ} \mathrm{C}$ for 45 seconds; and final extension at $72^{\circ} \mathrm{C}$ for 10 minutes. We purified PCR amplification products using the QIAquick PCR Purification Kit (QIAGEN, USA). We used the purified PCR products for library preparation and high-throughput sequencing on an Illumina HiSeq 1500 sequencer (Novogene Bioinformatics Technology, China).

2.3. Sequencing Data and Statistical Analyses. To analyze the high-throughput sequencing data, we performed comprehensive bioinformatic and statistical analyses as previously described $[30,31]$. First, we used FLASH v1.2.7 to assemble the sequence reads via overlap sequences of the paired-end reads after high-throughput sequencing. Then, we obtained high-quality reads after removing low-quality reads (Phred quality score $<\mathrm{Q} 30$ ), ambiguous bases, and adapter sequences. We used Trimmomatic v0.33 to trim adapters and UCHIME v4.2 to remove chimeric sequences [32]. We classified the remaining sequences into operational taxonomic units (OTUs) according to their identities. Sequences with $>97 \%$ identity were clustered into an OTU, and the relative abundances of OTUs were estimated. We used QIIME and UCLUST to select the most abundant sequence as a representative sequence for each OTU [33]. We classified each OTU according to the SILVA 16S rRNA gene reference alignment database and assigned each a taxonomic identity. We classified the taxon abundance of each sample according to phylum, class, order, family, and genus 
by using the RDP and GreenGene databases. We calculated UniFrac distance using phylogenetic information, which was based on the phylogeny inference of sequence alignment determined by PyNAST.

We determined alpha diversity from rarefied OTU tables by using mothur software, which included the Chao1, ACE, Shannon, Simpson, observed species (i.e., OTU number), and coverage indexes [34]. Meanwhile, to compare diversity between groups, we calculated beta diversity by using mothur software, namely, principal coordinate analysis (PCoA), based on unweighted and weighted UniFrac phylogenetic distance [35]. We statistically analyzed alpha diversity by using the Wilcoxon rank-sum test and beta diversity by using permutational multivariate analysis of variance (PERMANOVA) in the vegan package in $\mathrm{R}$ [30]. We performed the Wilcoxon rank-sum test to detect the significance of the relative abundance of gut microbiota between sample groups at the phylum, family, and genus levels; the results were corrected by false discovery rate (FDR). We used linear discriminant analysis effect size analysis to determine the bacterial taxa that differed significantly between groups [36]. The level of significance was set at $p<0.05$.

2.4. Immunohistochemistry. On the day of sacrifice, we weighed and deeply anaesthetized mice by intraperitoneal injection of $0.6 \%$ pentobarbital $(70 \mathrm{mg} / \mathrm{kg})$ before transcardially perfusing them with $4 \%$ paraformaldehyde (PFA) in phosphate-buffered saline (PBS, pH 7.4). Then, we excised the brains and postfixed them overnight in 4\% PFA before transferring them to $30 \%$ sucrose solution for cryoprotective procedures. We performed immunostaining on free-floating $20 \mu \mathrm{m}$ cryostat sections. We blocked sections in $10 \%$ goat serum in TBS-T (0.1\% Triton X-100, Sigma) for $1 \mathrm{~h}$ before incubation with anti-A $\beta_{17-24}(4 \mathrm{G} 8,1: 1,000$, Covance) and anti-Ibal (1:500, 019-19741, Wako) primary antibodies overnight at $4^{\circ} \mathrm{C}$. After washing with TBS-T, we incubated the sections with anti-mouse-Alexa 488- and anti-rabbitAlexa 568-conjugated secondary antibodies (1:1,000, Invitrogen) for $2 \mathrm{~h}$ at room temperature while protecting them from light. We counterstained the sections with DAPI ( $4^{\prime}$, 6-diamidino-2-phenylindole) and mounted them with Fluoromount-G (SouthernBiotech). We acquired images at $10 \mathrm{x}, 20 \mathrm{x}$, and $63 \mathrm{x}$ magnification using a Zeiss LSM 880 confocal microscope.

2.5. Quantification of A $\beta$ Plaque Burden and Iba $1^{+}$Microglia. We quantified A $\beta$ plaque staining by using the Analyze Particles tool in ImageJ software. For each section, we set intensity thresholds to eliminate background fluorescence and quantified 4 sections for each mouse $(n=4$ mice for each age group) throughout the study. The $\mathrm{A} \beta$ plaque load percentage was determined by dividing the total plaque area by the area of the cerebral cortical region of each section [37]. The percentage of $\mathrm{Ibal}^{+}$microglial immunoreactivity was calculated using a similar method (i.e., by using the Analyze Particles tool in ImageJ to measure the total $\mathrm{Ibal}^{+}$area and dividing it by the area of the cerebral cortex of each section) [38]. Plaque-localized microglial cell numbers in APP/PS1 mice were counted using ImageJ software and compared to those in an arbitrary location in the cortex of WT mice. Specifically, Iba1-positive somas colocalized with DAPI and within the $200 \mu \mathrm{m}$ vicinity of $\mathrm{A} \beta$ plaques were identified as plaque-localized microglia [27]. The number of activated microglia with deramified morphology around the plaque was quantified manually in each section [39]. Statistical analysis was performed by using GraphPad Prism software. To compare the $\mathrm{A} \beta$ plaque load among the 5 age groups $(1,2,3,6$, and 9 months) in APP/PS1 mice, we performed one-way ANOVA followed by the Bonferroni multiplecomparison post hoc analysis. For the comparisons of $\mathrm{Ibal}^{+}$ microglia between the WT and APP/PS1 mice at different time points, we applied two-way ANOVA followed by the Bonferroni multiple-comparison test. A $p$ value $<0.05$ was considered statistically significant.

\section{Results}

3.1. Characterization of Gut Microbiota Sequencing Data. To investigate how intestinal microbiota is regulated during AD development, we systematically examined age-related changes in the microbiota composition in APP/PS1 mice in comparison to age-matched WT littermates (Table 1). There was a total of 9,987,522 V3-V4 $16 \mathrm{~S}$ rRNA sequence reads from 203 samples, with an average of 47,738 sequence reads per sample. The average length of sequence reads was $315 \mathrm{bp}$ (Supplementary Figure S1). Sequences were clustered into OTUs according to sequence identity.

\subsection{Alterations in Gut Microbiome Composition in APP/PS1} Mice. To delineate the changes in gut microbiota with age, we compared microbial abundance at the phylum, class, order, family, and genus levels between the WT and APP/PS1 mouse feces. Across all age groups in both the WT and APP/PS1 mice, the dominant phyla were Firmicutes and Bacteroidetes (Figure 1(a)). The ratio of the abundance of Firmicutes to Bacteroidetes shifted with age; the changes were similar between the WT and APP/PS1 mice (Figure 1(b), Table 2). We observed similar trends in the top 2 bacterial taxa at the rank of class, order, family, and genus (Figures 1(c)-1(f)). To further characterize gut microbiota composition, we performed alpha diversity analysis, which measures the abundance and richness of OTUs within a given sample. In both the WT and APP/PS1 mice, the OTU number, ACE index, and Chaol index trended towards increased diversity and richness from 1 to 6 months and a decline in diversity at 9 months (Figures 2(a)-2(c)). However, compared to age-matched WT mice, the microbiota of 9-month-old APP/PS1 mice exhibited greater richness and diversity with respect to the OTU number, ACE index, and Chaol index (Wilcoxon rank-sum test; OTU number: $p=0.021$; ACE: $p=0.008$; Chaol: $p=0.012$ ). Surprisingly, at 2 months, APP/PS1 mice also exhibited greater richness with respect to the Chaol index than WT mice (Wilcoxon rank-sum test; Chaol: $p=0.045$ ) (Table 3). There were no significant differences in the Simpson, Shannon, or coverage indexes between the WT and APP/PS1 mice at any age (data not shown). 

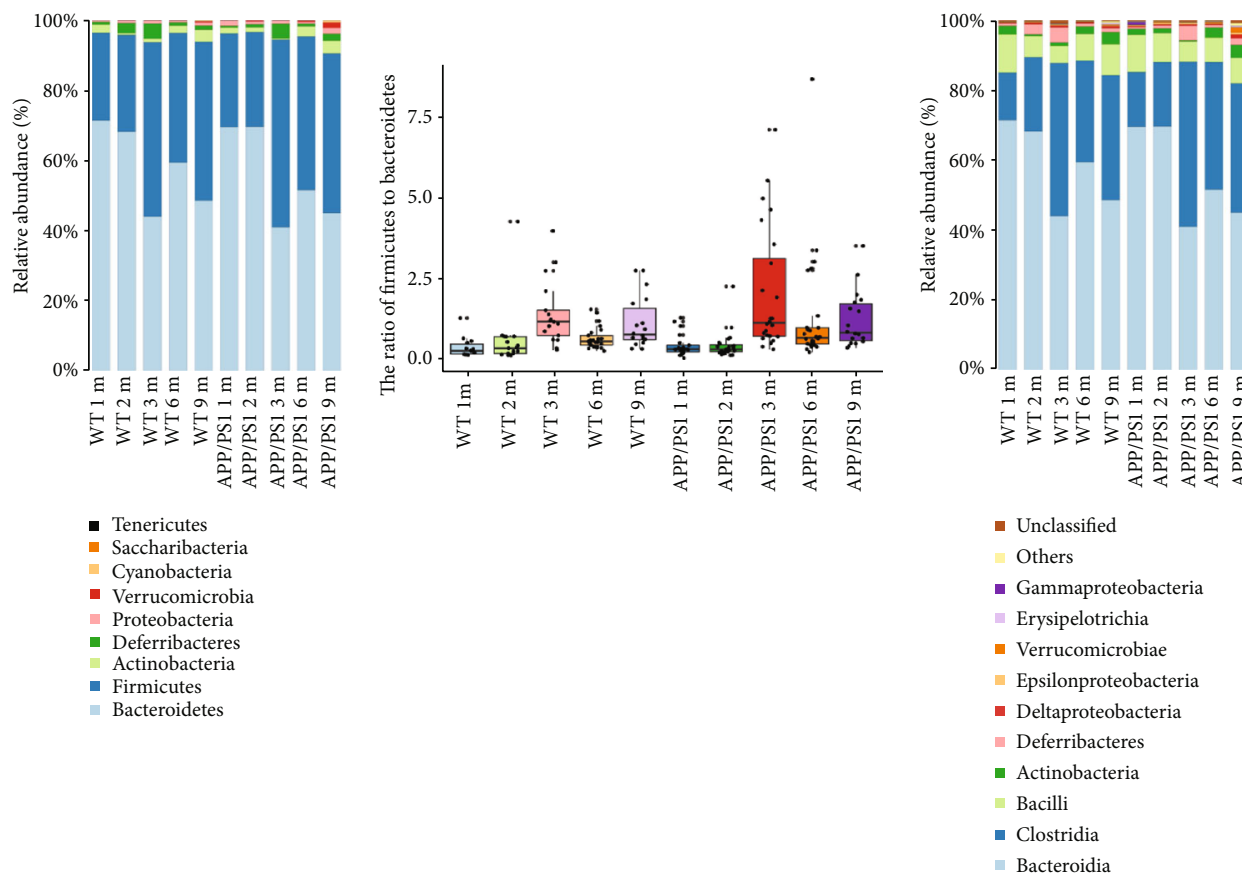

(a)

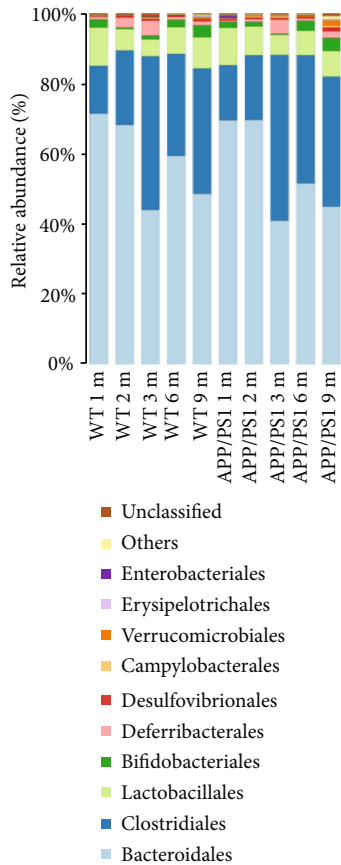

(d) (b)

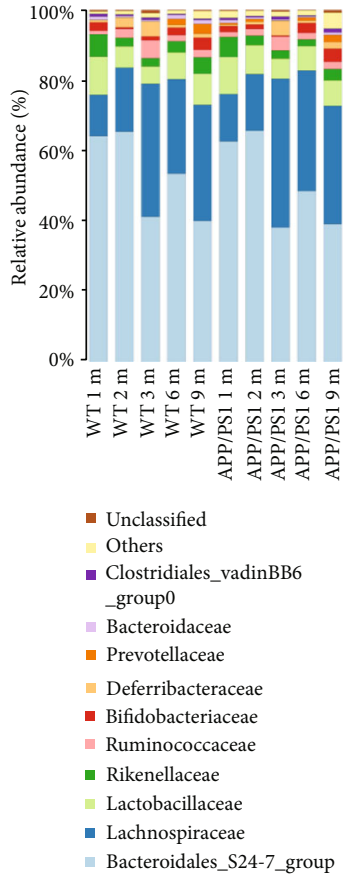

(e) (c)

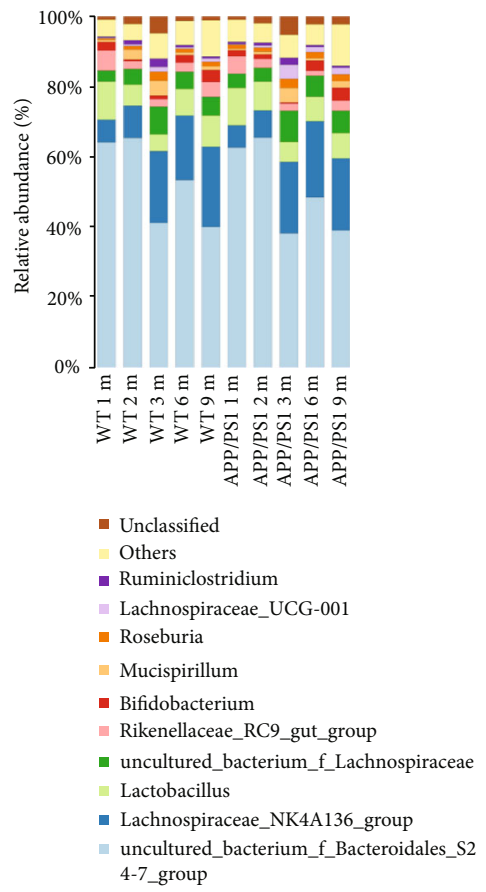

(f)

Figure 1: Changes in microbe composition in mouse feces along development and aging. (a) The relative abundance of top microbes in mouse feces is shown at the rank of phylum. (b) The ratio of the abundance of Firmicutes to Bacteroidetes in all groups. (c-f) Profiles of the microbes in mouse feces at the rank of (c) class, (d) order, (e) family, and (f) genus. $1 \mathrm{~m}, 2 \mathrm{~m}, 3 \mathrm{~m}, 6 \mathrm{~m}$, and $9 \mathrm{~m}$ indicate mice at 1,2 , 3,6 , and 9 months of age, respectively.

Meanwhile, analysis of beta diversity using PCoA and unweighted UniFrac demonstrated significant differences in microbiome composition between the WT and APP/PS1 mice at 2 and 6 months (PERMANOVA; WT vs. APP/PS1 at 2 months: $p=0.035$; WT vs. APP/PS1 at 6 months: $p=0.045$ ) (Figures $2(\mathrm{~d})-2(\mathrm{~h})$, Table 4). These results reveal that the composition and community structures of the gut microbiota are altered in APP/PS1 mice starting from an early age. To provide a more intuitive representation of the differences in the distribution of OTU abundance between the WT and APP/PS1 mice at each age, we plotted cluster heatmaps of differentially abundant taxa at the OTU level 
TABLE 2: Ratio of the abundance of Firmicutes to Bacteroidetes.

\begin{tabular}{lccccc}
\hline Mouse group & 1 month & 2 months & 3 months & 6 months & 9 months \\
\hline WT mice & 0.35 & 0.40 & 1.13 & 0.62 & 0.93 \\
APP/PS1 mice & 0.38 & 0.39 & 1.30 & 0.85 & 1.01 \\
\hline
\end{tabular}

WT: wild type.

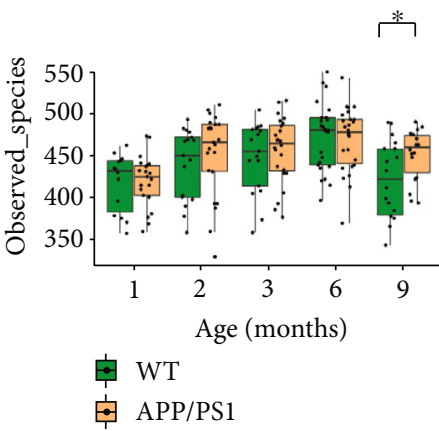

(a)

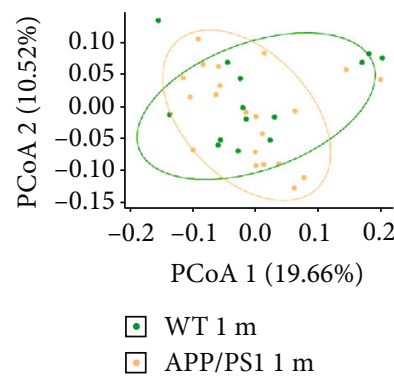

(d)

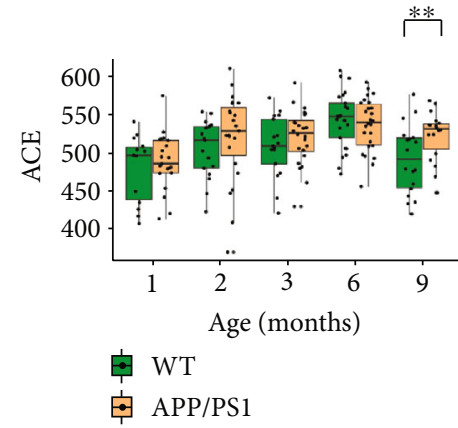

(b)

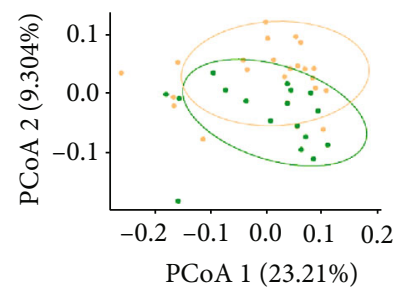

$\square$ WT $2 \mathrm{~m}$

$\square$ APP/PS1 $2 \mathrm{~m}$

(e)

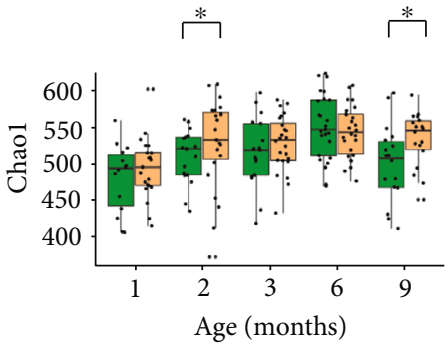

它 WT

官 APP/PS1

(c)

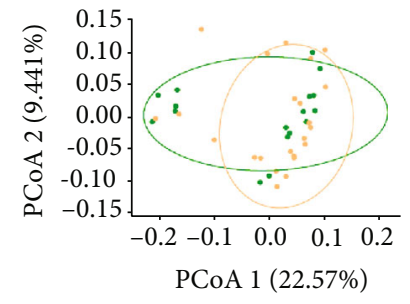

$\square$ WT $3 \mathrm{~m}$

$\square$ APP/PS1 3m

(f)

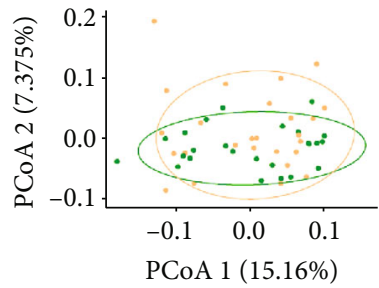

$\square$ WT $6 \mathrm{~m}$

APP/PS1 $6 \mathrm{~m}$

(g)

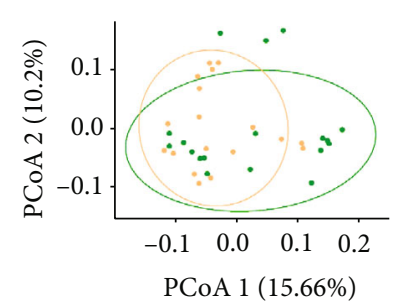

$\square$ WT $9 \mathrm{~m}$

$\square$ APP/PS1 $9 \mathrm{~m}$

(h)

FIGURE 2: Age-dependent alteration of gut microbiome composition in Alzheimer's disease mice. (a-c) Alpha diversity was determined on the basis of the numbers of observed species as well as the ACE and Chaol indexes. (d-h) Principal coordinate analysis (PCoA) based on unweighted and weighted UniFrac phylogenetic distance was compared between the wild-type (WT) and APP/PS1 mice at different ages. ${ }^{*} p<0.05,{ }^{* *} p<0.01 .1 \mathrm{~m}, 2 \mathrm{~m}, 3 \mathrm{~m}, 6 \mathrm{~m}$, and $9 \mathrm{~m}$ indicate mice at $1,2,3,6$, and 9 months of age, respectively.

(Supplementary Figure S2). The differential abundance of OTUs revealed that compared to WT mice, APP/PS1 mice had significantly altered gut microbiome composition across all age groups.

3.3. Differentially Represented Bacterial Taxa in APP/PS1 Mice. Linear discriminant analysis effect size analysis showed that the gut microbial communities in both the WT and APP/PS1 mice changed dynamically with age, with most dif- ferentially abundant taxa being present in 9-month-old mice $(p<0.05) \quad$ (Supplementary Figure S3). We subsequently applied multiple comparisons to analyze microbial taxa that were differentially represented between the WT and APP/PS1 mice at the phylum and family levels (Figure 3) and genus level Figure 4, summarized in Supplementary Table S2). Among the identified taxa, we focused on the taxa with occupancies $\geq 0.01 \%$ at each taxon level [25]. Compared to age-matched WT mice, 1-month-old APP/PS1 
TABLE 3: Comparison of alpha diversity between the wild-type and APP/PS1 mice.

\begin{tabular}{lccc}
\hline & ACE $(p$ value $)$ & Chao1 $(p$ value $)$ & OTU $(p$ value $)$ \\
\hline WT-APP/PS1 $1 \mathrm{~m}$ & 0.479859 & 0.277845 & 0.433141 \\
WT-APP/PS1 2 m & 0.06619 & $0.044915^{*}$ & 0.11163 \\
WT-APP/PS1 3 m & 0.173755 & 0.241569 & 0.280179 \\
WT-APP/PS1 6 m & 0.302173 & 0.282751 & 0.481211 \\
WT-APP/PS1 9 m & $0.008454^{* *}$ & $0.011844^{*}$ & $0.021394^{*}$ \\
\hline
\end{tabular}

OTU: operational taxonomic unit; WT: wild type. Alpha diversity was assessed by the Wilcoxon rank-sum test; ${ }^{*} p<0.05,{ }^{* *} p<0.01$. WT-APP/PS1 $1 \mathrm{~m}$, WT-APP/PS1 $2 \mathrm{~m}$, WT-APP/PS1 $3 \mathrm{~m}$, WT-APP/PS1 $6 \mathrm{~m}$, and WT-APP/PS1 $9 \mathrm{~m}$ show comparisons between WT mice and APP/PS1 transgenic mice at $1,2,3,6$, and 9 months of age, respectively.

TABle 4: Comparison of beta diversity between the wild-type and APP/PS1 mice by Pairwise.adonis (unifrac_1, detail).

\begin{tabular}{lccccc}
\hline & F.Model & $R^{2}$ & $p$ value & Adjusted $p$ value & Significance \\
\hline WT-APP/PS1 1 m & 0.7535174 & 0.02232412 & 0.817 & 0.817 & 0.035 \\
WT-APP/PS1 2 m & 1.861077 & 0.04668909 & 0.035 & 0.052 & $\mathrm{n}$ \\
WT-APP/PS1 3 m & 1.614989 & 0.03976336 & 0.052 & 0.045 & 0.045 \\
WT-APP/PS1 6 m & 1.523908 & 0.03016212 & 0.246 & $\mathrm{n}$ \\
WT-APP/PS1 9 m & 1.171244 & 0.03330119 & 0.246 & 0 \\
\hline
\end{tabular}

ns: not significant; WT: wild type. Beta diversity was assessed by PERMANOVA; ${ }^{*} p<0.05$. WT-APP/PS1 $1 \mathrm{~m}, \mathrm{WT}-\mathrm{APP} / \mathrm{PS} 12 \mathrm{~m}, \mathrm{WT}-\mathrm{APP} / \mathrm{PS} 13 \mathrm{~m}$, WT-APP/PS1 $6 \mathrm{~m}$, and WT-APP/PS1 $9 \mathrm{~m}$ show comparisons between WT mice and APP/PS1 transgenic mice at 1, 2, 3, 6, and 9 months of age, respectively.

mice exhibited a significantly greater relative abundance of Enterobacteriaceae (phylum Proteobacteria) (Figure 3(a)). In addition, compared to age-matched WT mice, the relative abundance of phylum Verrucomicrobia and family Verrucomicrobiaceae was significantly higher in APP/PS1 mice at 2, 6, and 9 months, respectively (Figures 3(b)-3(g)). Moreover, the relative abundance of phyla Actinobacteria and Proteobacteria increased significantly in APP/PS1 mice at 2 and 9 months, respectively (Figures 3(b) and 3(f)). Besides Verrucomicrobiaceae, other bacterial families such as Prevotellaceae, Erysipelotrichaceae, and Bifidobacteriaceae were more abundant in APP/PS1 mice than in WT mice at 2 months (Figure 3(c)). There were no significant differences in microbiota composition between the 3-month-old APP/PS1 and WT mice at the phylum or family level (data not shown). Compared to age-matched WT controls, 6month-old APP/PS1 mice exhibited significantly greater abundance of Enterobacteriaceae and Verrucomicrobiaceae as well as significantly lower abundance of Bacteroidaceae and Rikenellaceae (Figure 3(e)). By 9 months, the abundance of Verrucomicrobiaceae, Desulfovibrionaceae, Clostridiales_ vadinBB60_group, and an unclassified taxon increased significantly in APP/PS1 mice, whereas that of Carnobacteriaceae decreased (Figure 3(g)).

At the genus level, compared to age-matched WT mice, APP/PS1 mice exhibited significantly greater abundance of Akkermansia (phylum Verrucomicrobia) at 2, 6, and 9 months, respectively (Figures 4(a), 4(c), and 4(d)). In addition, at 2 months, Prevotellaceae_UCG-001, Bifidobacterium, Erysipelatoclostridium, Allobaculum, and Alloprevotella were more abundant in APP/PS1 mice than WT mice (Figure 4(a)). There were no 5significant differences in microbiota composition at the genus level between the
APP/PS1 and WT mice at 1 month (data not shown). At 3 months, compared to age-matched WT mice, Lachnospiraceae_UCG_001, a member of Lachnospiraceae in phylum Firmicutes, was more abundant in APP/PS1 mice, whereas the abundance of Ruminococcaceae_UCG_010 and Ruminiciostridium_5 (both phylum Firmicutes) was lower in APP/PS1 mice (Figure 4(b)). At 6 months, compared to WT mice, Escherichia-Shigella, Akkermansia, Blautia, Turicibacter, Tyzzerella, Family_XIII_UCG-001, and an unclassified taxon were more abundant in APP/PS1 mice, whereas Bacteroides and Rikenellaceae_RC9_gut_group were less abundant in APP/PS1 mice (Figure 4(c)). Finally, at 9 months, compared to WT mice, Desulfovibrio, Akkermansia, uncultured_bacterium_f_Clostridiales_vadinBB60_group, Lachnospiraceae_UCG-001, Ruminococcus_1, and an unclassified genus were more abundant in APP/PS1 mice, whereas Atopostipes, Eubacterium_nodatum_group, Alistipes, and Lachnospiraceae_UCG-006 were less abundant in APP/PS1 mice (Figure $4(\mathrm{~d})$ ). These results collectively indicate that the abundance of several gut bacteria begins to differ significantly between the WT and APP/PS1 mice during the early stages of $\mathrm{AD}$ development and that these differences increase with age.

3.4. Association of Microglia with A $\beta$ Plaques in the Cerebral Cortex in APP/PS1 Mice. To further examine the relationships between gut microbiota alterations and neuroinflammatory states in $\mathrm{AD}$ pathology, we analyzed the reactive gliosis surrounding $\mathrm{A} \beta$ plaques across all age groups. Given that Iba1 is commonly used as a general microglial marker in the brain, we performed the immunofluorescence staining with anti-Ibal together with $\mathrm{A} \beta$ antibodies to reveal the distribution of microglia in relation to $\mathrm{A} \beta$ plaques in APP/PS1 mice. In 1 - and 2-month-old APP/PS1 mice, there were 


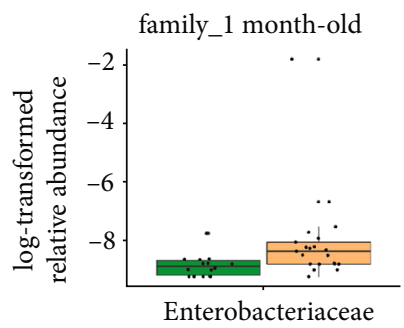

$\square$ WT 9 m

$\square$ APP/PS1 $9 \mathrm{~m}$

(a)

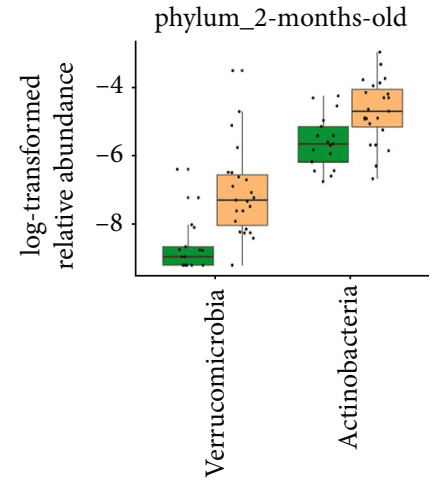

家 WT

官 APP/PS1

(b)

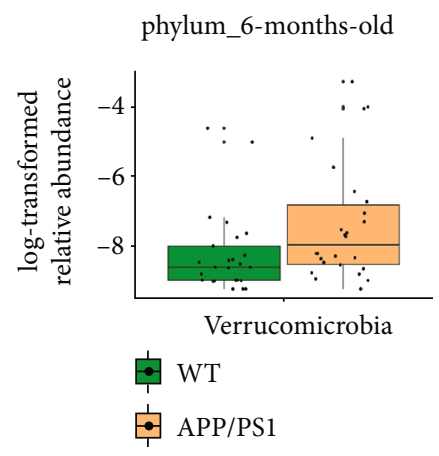

审 APP/PS1

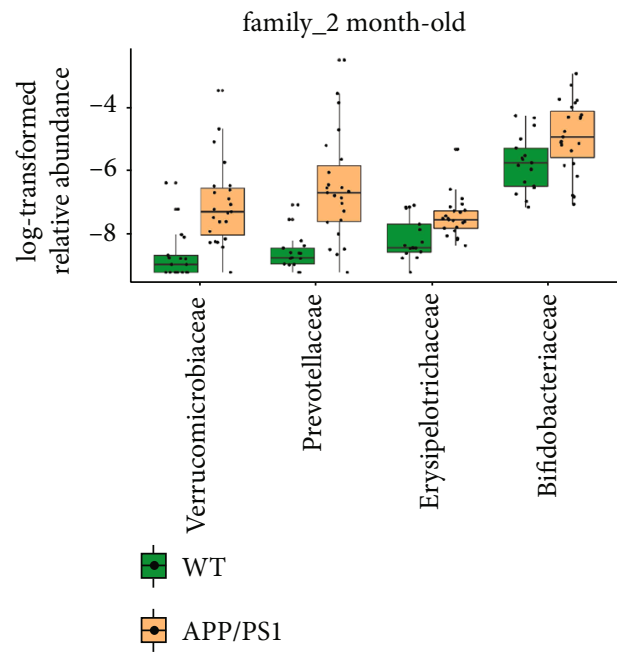

(c)

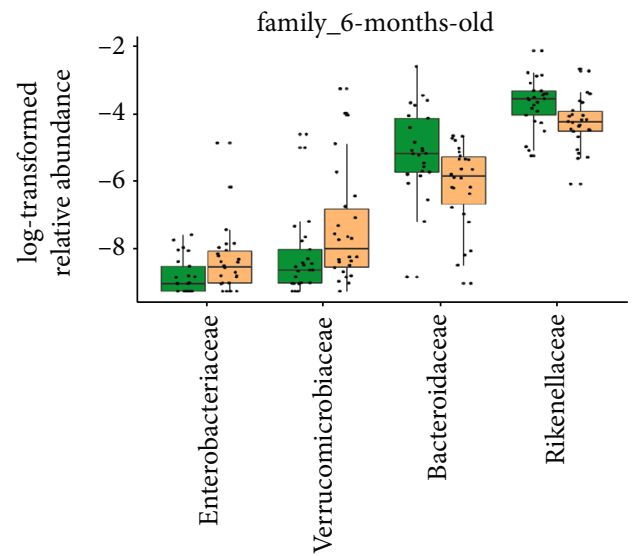

年 WT

舟 APP/PS1

(d)

(e)

Figure 3: Continued. 

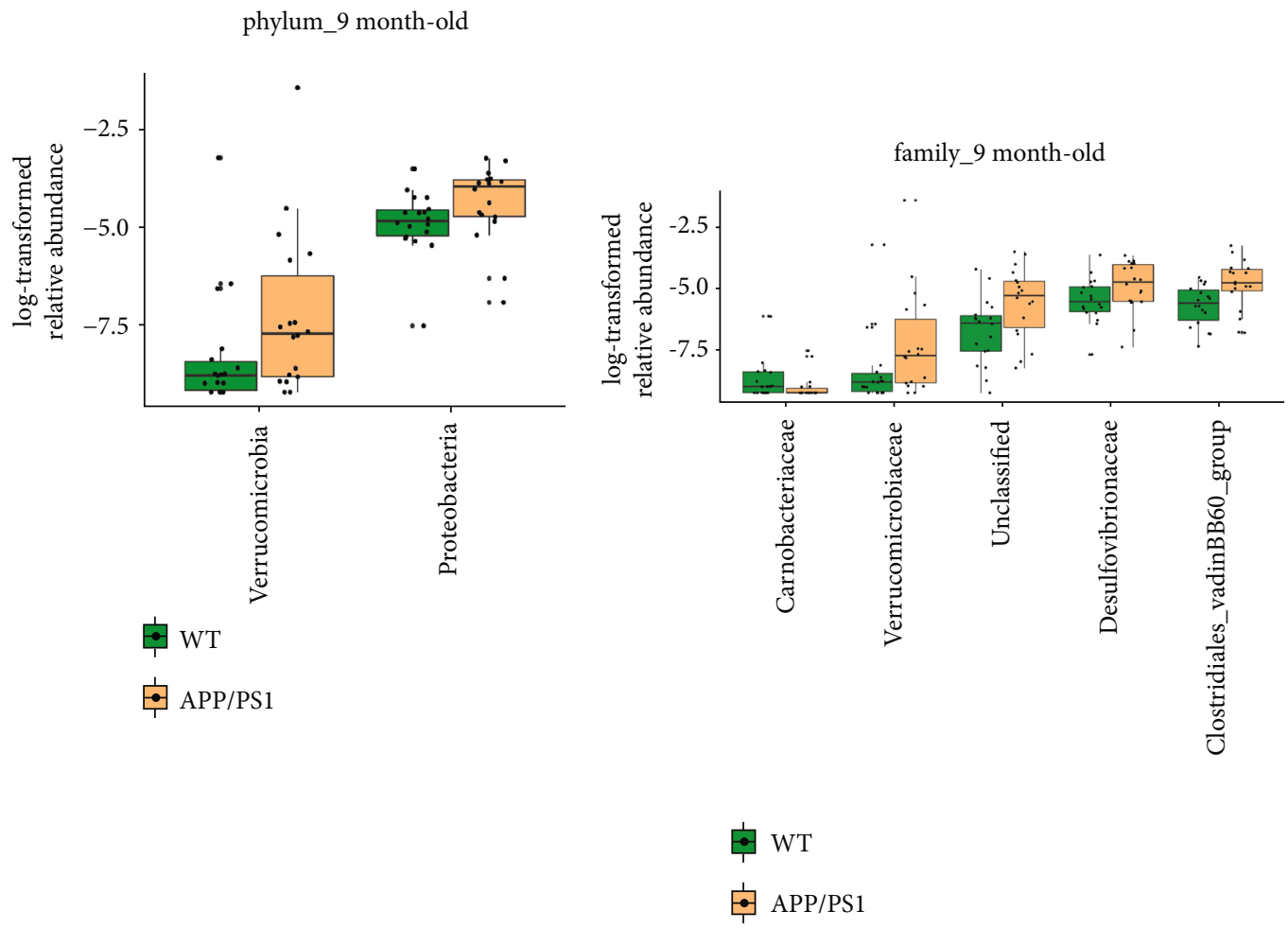

(f)

(g)

FIgURE 3: Variation of bacterial composition at the phylum and family levels in Alzheimer's disease mouse model feces. Data are presented as log-transformed relative abundance. $(\mathrm{a}-\mathrm{g})$ There were significant differences in the bacterial phyla and families with relative abundance $\geq 0.01 \%$ between the APP/PS1 and wild-type (WT) mice at $1,2,6$, and 9 months of age, respectively ( $p<0.05$, false discovery rate- (FDR-) corrected).

no $\mathrm{A} \beta$ plaques in the cerebral cortex, and the microglia were evenly distributed (Figures $5(\mathrm{k})$ and 5(l); highermagnification images in Figures 5(p) and 5(q), respectively). Very little $\mathrm{A} \beta$ staining was detected in the cortex in 3-monthold APP/PS1 mice, whereas obvious $\mathrm{A} \beta$ plaques were observed at 6 and 9 months, with the microglia clustered around them (Figures 5(m)-5(o); higher-magnification images in Figures 5(r)-5(t), respectively). On the other hand, there were no $\mathrm{A} \beta$ plaques in age-matched WT mice, and microglia were evenly distributed in the cortex in WT mice at all ages (Figures 5(a)-5(e); higher-magnification images in Figures 5(f) $-5(j)$, respectively). Quantification of Iba1 immunoreactivity confirmed a significant increase in activated microglia and plaque-localized and deramified microglia in the cortex in APP/PS1 mice at 6 and 9 months (Figures 5(u)-5(w)). Given that we identified significant differences in microbial abundance between APP/PS1 mice and wild-type control mice at the early stages (i.e., 1-3 months) when $\mathrm{A} \beta$ plaque accumulation and microglial activation have not occurred in the cerebral cortex, our results suggest that alteration of the gut microbiome profile occurs prior to amyloidosis and plaque-localized microglial activation in the brain of $\mathrm{AD}$ model mice.

\section{Discussion}

Recent studies have unveiled a link between gut microbiota homeostasis and $\mathrm{AD}$ progression. However, the changes in microbiota profiles during $\mathrm{AD}$ development, especially in the early stages, are unclear. Therefore, in the present study, to examine the association between gut microbiota composition and the progression of AD pathology, we compared the fecal microbiome of WT and AD model mice (i.e., APP/PS1 mice) starting from an early age. We observed a shift in gut microbiota composition in the young adult WT and $\mathrm{APP} / \mathrm{PS} 1$ mice as well as a tendency of microbial richness and diversity to decline in older mice. Our results are consistent with previous results showing that APP/PS1 mice exhibit greater richness in gut microbiota than WT mice by the Chaol index, without a significant difference in the Simpson diversity between the 2 groups [24]. Microbial diversity usually increases after birth, and microbial composition changes gradually during late childhood, adolescence, and adulthood $[40,41]$. Furthermore, reduced microbial diversity is associated with aging or poorer health conditions in humans [4244]. The loss of diversity and decreased temporal compositional stability in elderly people are related to general health as well as to confounding factors such as diet and constrained lifestyles [45]. Recent studies showed that gut microbiota composition in $\mathrm{AD}$ patients differs from that in normal controls recruited from the same geographical area and shared dietary food habits; this indicates that the alteration of gut microbiota in $\mathrm{AD}$ patients may not be due to diet or comorbidities [23]. Our results consistently show a trend towards increased diversity and richness from 1 to 6 months and decreased diversity at 9 months in both the WT and APP/PS1 


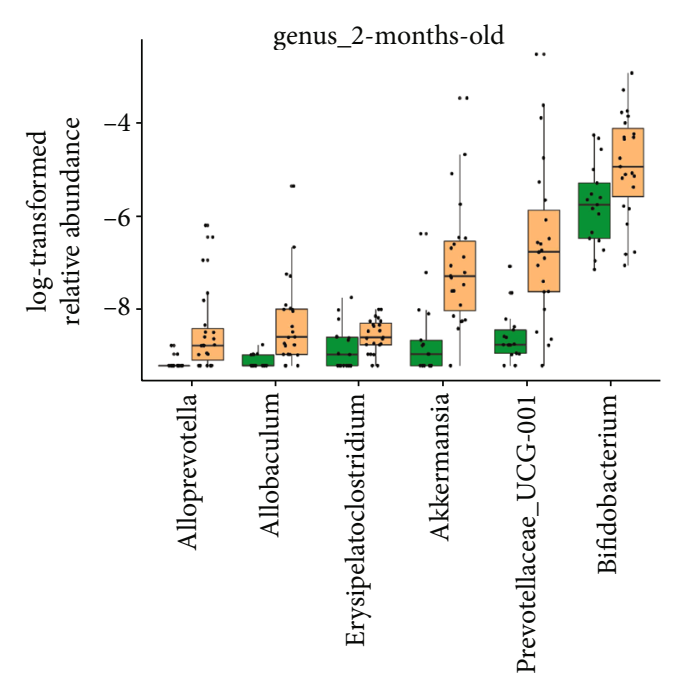

WT

由 APP/PS1

(a)

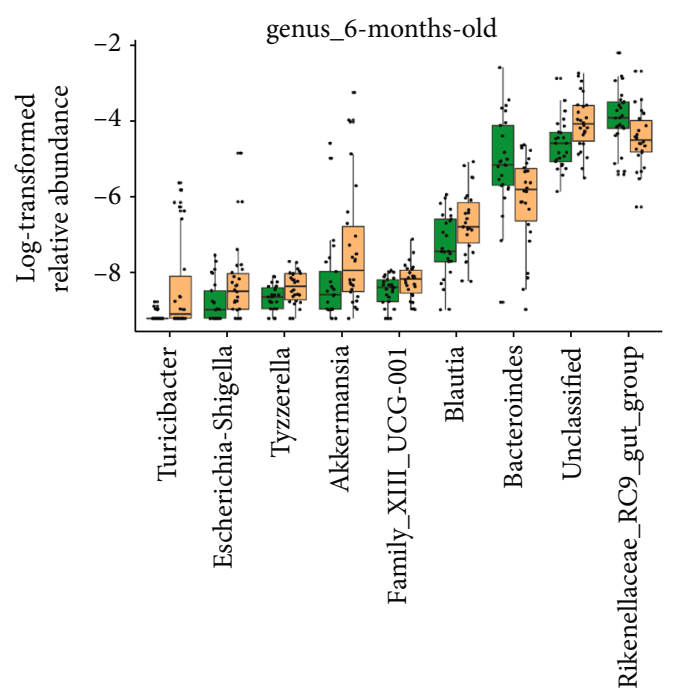

부 APP/PS1

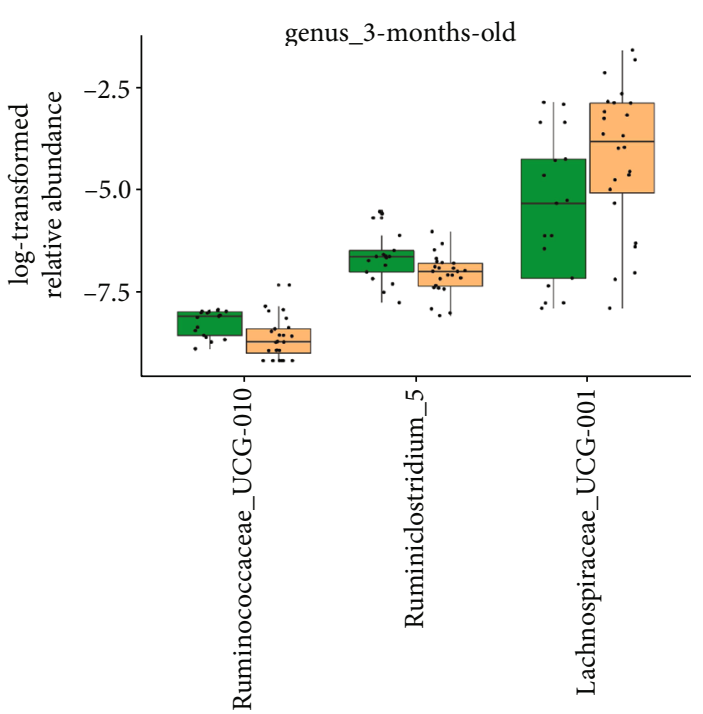

审 WT

由 APP/PS1

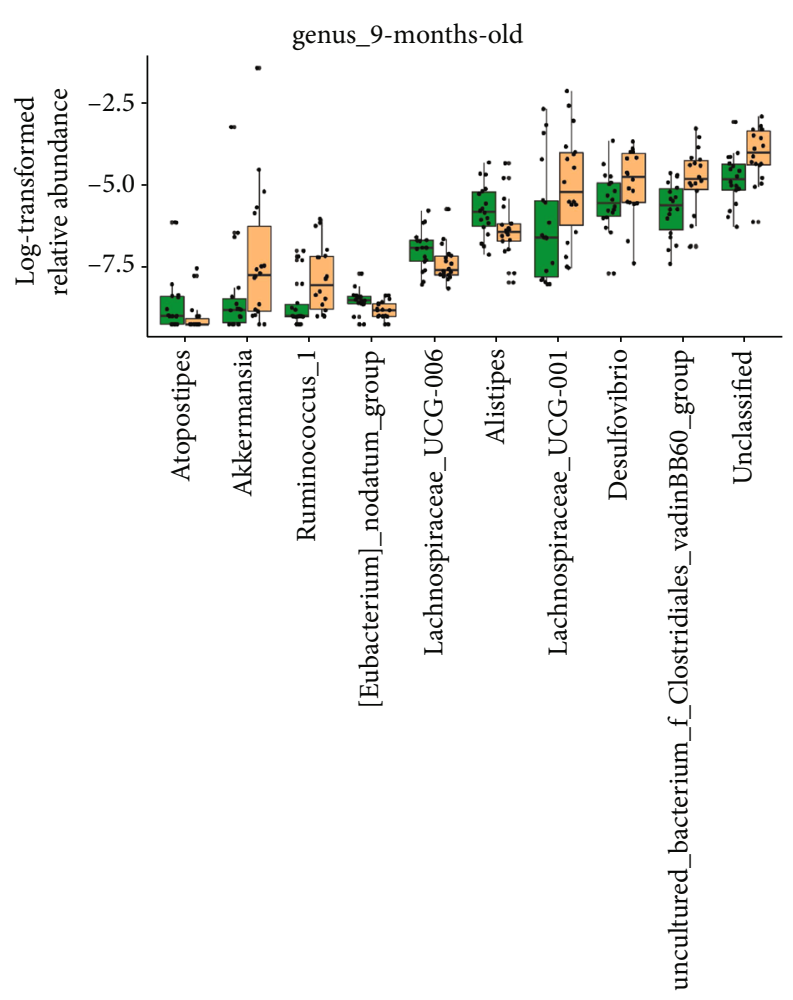

田 WT

甶 APP/PS1

(c)

(d)

Figure 4: Variation of bacterial composition at the genus level in Alzheimer's disease mouse model feces. Data are presented as log-transformed relative abundance. (a-d) There were significant differences in the bacterial genera with relative abundance $\geq 0.01 \%$ between the APP/PS1 and wild-type (WT) mice at 2, 3, 6, and 9 months of age, respectively ( $p<0.05$, FDR-corrected).

mice, indicating aging as a general factor influencing microbial composition. However, our results further demonstrate that the progression of $\mathrm{AD}$ is associated with additional changes in the abundance of inflammation-related gut microbiome composition against a background of agedependent alteration of diversity. This finding indicates that the condition of $\mathrm{AD}$ may impose extra pressure on the regulation of microbial composition and abundance during aging. 


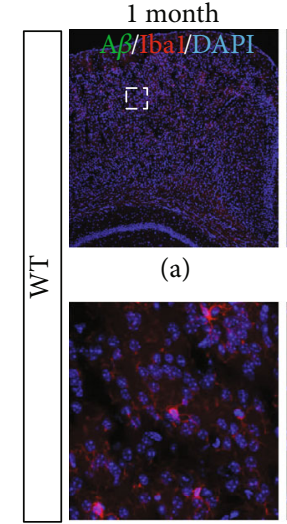

(f)

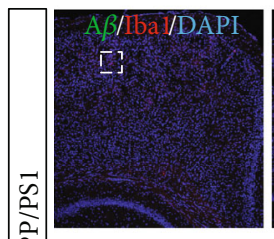

$(\mathrm{k})$

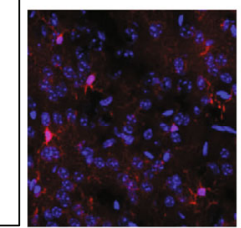

(p)
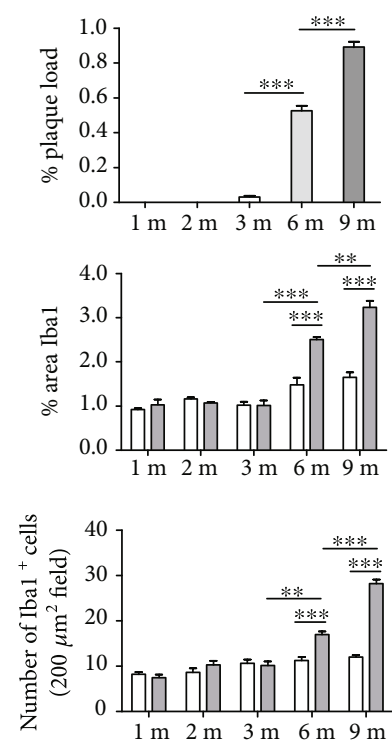

$\square \mathrm{WT}$

$\square \mathrm{APP} / \mathrm{PS}$

(u)
2 months

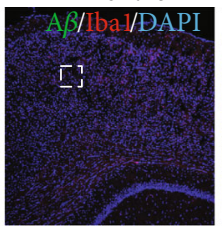

(b)

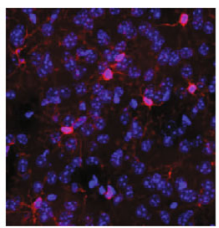

(g)

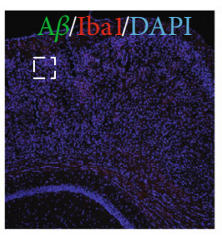

(l)

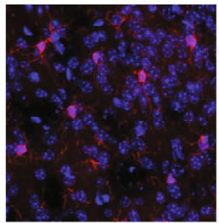

(q)

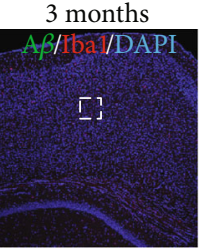

(c)

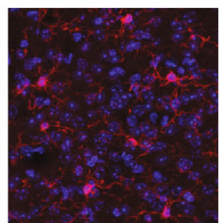

(h)

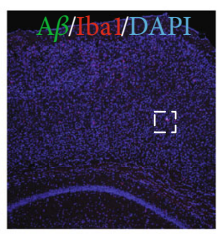

(m)

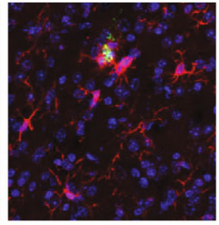

(r)

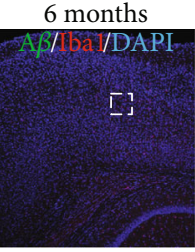

(d)

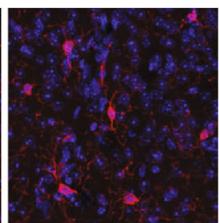

(i)

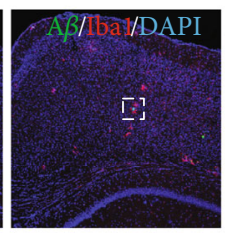

(n)

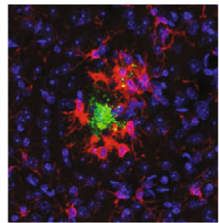

(s)
9 months

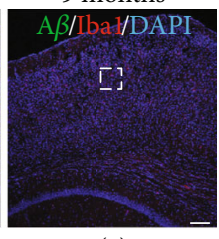

(e)

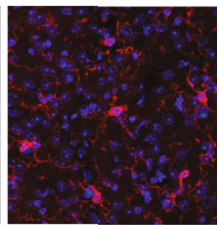

(j)

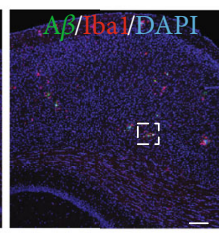

(o)

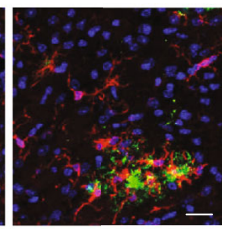

(t)

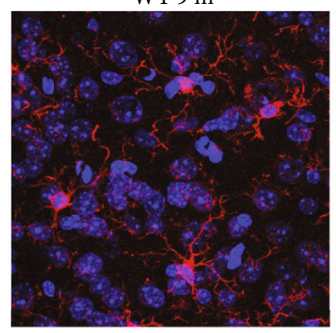

APP/PS1 9 m

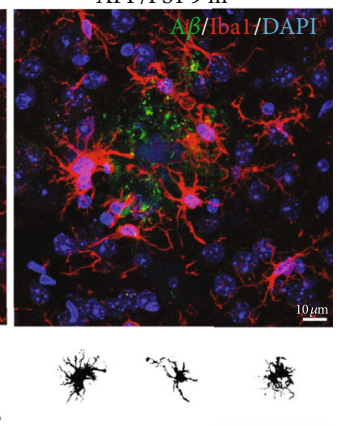

(v)

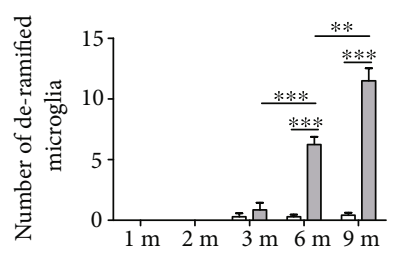

(w)

FIGURE 5: Increased amyloidosis and plaque-localized microglial activation in Alzheimer's disease mouse models during aging. Brain sections derived from the representative wild-type (WT) and APP/PS1 mice at 1,2,3,6, and 9 months of age were immunolabeled with anti-Iba1 (red) and amyloid-beta (A $\beta$, green) antibodies and counterstained with DAPI. (a-e) and (k-o) are $z$-stack maximum projection images of the WT and APP/PS1 mice, respectively; $(\mathrm{f}-\mathrm{j})$ and $(\mathrm{p}-\mathrm{t})$ are higher-magnification images of the areas denoted by white rectangles, respectively. (u) Quantification of the amyloid-beta $(\mathrm{A} \beta)$ plaque load, Ibal immunoreactivity, and plaque-localized $\mathrm{Iba1}^{+} \mathrm{microglia}$ in APP/PS1 mice at 1 ,

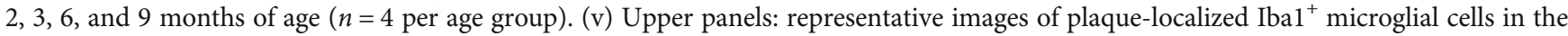
cortex in 9-month-old APP/PS1 mice; lower panels: representative images showing the morphology of microglial cells. (w) Quantification of deramified microglia in APP/PS1 mice at 1, 2, 3, 6, and 9 months of age versus their WT controls ( $n=4$ per age group). Data are presented as mean \pm SEM. ${ }^{* *} p<0.01,{ }^{* * *} p<0.001$. Scale bars: $100 \mu \mathrm{m}(\mathrm{a}-\mathrm{e}, \mathrm{k}-\mathrm{o}), 25 \mu \mathrm{m}(\mathrm{f}-\mathrm{j}, \mathrm{p}-\mathrm{t})$, and $10 \mu \mathrm{m}(\mathrm{v})$. 
We also identified several bacterial taxa whose abundance differed between the WT and APP/PS1 mice at different ages. Notably, compared to WT mice, significant differences in microbial abundance emerged at early ages in APP/PS1 mice (i.e., 1-3 months), while only obvious A $\beta$ plaques and plaque-localized microglial activation were observed in the cerebral cortex in APP/PS1 mice older than 6 months. These findings indicate that the changes in microbial composition occur prior to the detection of obvious amyloidosis in the cerebral cortex in APP/PS1 mice. Moreover, we found that the differences in bacterial taxa between the WT and APP/PS1 mice increased with age, specifically at 6 and 9 months, which coincides with the progression of amyloid plaque formation and microglial activation in the APP/PS1 mouse brain. These findings suggest that gut microbial composition might be associated with AD pathogenesis and that such alterations might serve as potential diagnostic biomarkers and/or therapeutic targets for $\mathrm{AD}$. There are some reports on gut microbiota composition in the APP/PS1 mouse model. However, as most of these studies focus on microbial changes in adult mice [25], there is a lack of evidence regarding whether and how the microbiota is altered in early AD. Previous longitudinal studies have used different time points and only revealed the changes of the gut microbiome at later stages without direct comparison to amyloidosis or microglial activation $[24,26]$. Our results are consistent with previous data that the gut microbial composition in $\mathrm{AD}$ mice shifts towards an inflammation-related bacterial profile in the feces of APP/PS1 mice as compared to their age-matched WT controls [24-26]. We also observe higher abundance of Escherichia-Shigella in our study, which was identified in AD patients [2] but has not been reported in other studies with APP/PS1 mice.

The inflammatory-infectious hypothesis of AD has elucidated the essential role of gut microbiota in the initiation of systemic inflammation, which leads to neuroinflammatory responses in the brain [46]. The gut microbiota might regulate the development and maturation processes of microglia and astrocytes as well as peripheral immune responses; in turn, the activation of innate immune responses might modulate the homeostasis of intestinal bacterial communities [47-49]. For example, Proteobacteria are implicated in immunological and inflammatory reactions and act as a microbial signature of dysbiosis in disease states [50]. Accordingly, altered Proteobacteria composition has been found in patients with $\mathrm{AD}$, irritable bowel syndrome, or Parkinson's disease $[22,51,52]$. Similarly, in the present study, the abundance of Enterobacteriaceae, a bacterial taxon belonging to Proteobacteria, was elevated in APP/PS1 mice as early as 1 month old. Meanwhile, elevated abundance of Enterobacteriaceae has been reported in the feces of children with autism and patients with Parkinson's disease, suggesting that these bacteria might be involved in CNS disorders [5356]. Enterobacteriaceae includes many pathogenic bacteria such as Escherichia coli and Shigella spp., which can produce endotoxins that are released into the bloodstream and consequently induce systemic inflammatory responses [57-59]. Our results indicate that young APP/PS1 mice have an aberrant gut microbial composition at the age when $\mathrm{A} \beta$ deposits have not yet developed in the brain. Moreover, the abundance of Proteobacteria was elevated in APP/PS1 mice at later ages; specifically, we observed increases in EscherichiaShigella at 6 months and Desulfovibrio at 9 months. Recent studies show that $\mathrm{A} \beta$ protects against microbial infection in the $5 x F A D$ transgenic mouse model, in the nematode Caenorhabditis elegans, and in cultured cell models of $\mathrm{AD}$ [4, 60]. Concordantly, a cohort study found that compared to both elderly healthy controls and patients with cognitive impairment without brain amyloidosis, the abundance of Escherichia-Shigella in the fecal microbiome was higher in elderly people with cognitive impairment and brain amyloidosis [2]. The same study further demonstrated that the abundance of Escherichia-Shigella was positively correlated with levels of proinflammatory cytokines such as IL-1 $\beta$, CXCL2, and NLRP3. Thus, these findings raise the possibility that bacterial infection might induce $\mathrm{A} \beta$ accumulation and promote the chronic inflammatory responses that drive $\mathrm{AD}$ pathogenesis [4, 28, 29]. Nevertheless, further investigation is required to determine whether Escherichia-Shigella is a risk factor for $\mathrm{AD}$ and how these bacteria trigger the inflammatory and pathologic processes.

In the present study, Verrucomicrobia increased consistently in APP/PS1 mice relative to age-matched WT mice at various ages, which was reflected by increased abundances of Verrucomicrobiaceae and Akkermansia at the family and genus levels, respectively. In addition, there was a trend towards elevated abundances of Verrucomicrobia and Akkermansia in older mice irrespective of genotype, suggesting that these bacterial taxa generally expand with age. Intriguingly, these differences were not observed in the 3-monthold groups. Indeed, the ratio of the abundance of Firmicutes to Bacteroidetes increased dramatically from 2 to 3 months and then declined at 6 months in both the WT and APP/PS1 mice, which indicates a special time point when the microbial community changes at the age of 3 months. This phenomenon may explain why few microbial taxa were detected between the 2 groups at this age. The abundance of Verrucomicrobia was recently reported to increase dramatically in older APP/PS1 mice, which suggests a potential association between these bacteria and AD progression [24]. Akkermansia muciniphila, the single known species within its genus, is a mucin-degrading bacterium that has attracted considerable attention in recent years because of its immunomodulatory effects on PD-1-based antitumor immunotherapy [61]. Accordingly, decreased Akkermansia abundance is associated with increased risks of inflammatory bowel disease, obesity, and type 2 diabetes [62, 63]. Furthermore, patients with advanced dementia and Clostridium difficile colonization, colon cancer, or multiple sclerosis exhibit an elevated abundance of Akkermansia [64-66]. Together with our current findings, these studies collectively indicate that Akkermansia has physiological significance in both healthy and disease conditions. Nevertheless, further studies are necessary to identify how Akkermansia modulates the immune system during $\mathrm{AD}$ progression.

Furthermore, recent evidence suggests that the metabolic byproducts of microbes, such as short-chain fatty acids, might be involved in the modulation of the peripheral and 
central immune pathways and might thus influence brain functions $[67,68]$. In the present study, APP/PS1 mice exhibited a significant increase in the abundance of Blautia at 6 months, when distinctive $\mathrm{AD}$ pathologic characteristics such as $\mathrm{A} \beta$ deposition are present in the brain. As a genus within family Lachnospiraceae in phylum Firmicutes, Blautia degrades polysaccharides into short-chain fatty acids that the host can use as an energy supply [69]. AD patients are reported to have an increased abundance of Blautia, which is correlated with more severe $\mathrm{AD}$ pathology, namely, lower levels of $\mathrm{A} \beta_{42} / \mathrm{A} \beta_{40}$ and higher levels of $p$-tau and $p$-tau/A $\beta_{42}$ in the cerebrospinal fluid [22]. Another study focusing on microbiota-derived extracellular vesicles in the blood also reports substantial elevation of Blautia abundance in APP/PS1 mice [70]. Moreover, an aberrant abundance of Blautia has been observed in other CNS disorders that share many characteristics with AD, namely, Parkinson's disease and multiple sclerosis $[52,71]$. These findings collectively suggest that Blautia might be a microbial indicator of disease state in certain CNS disorders and might thus serve as a potential biomarker for the evaluation of $\mathrm{AD}$ therapies.

Besides potential associations with disease states, alterations in the composition of the intestinal microbiome caused by probiotics or beneficial bacteria can confer health benefits to the host [72-74]. AD patients are reported to exhibit a decreased abundance of Actinobacteria, particularly Bifidobacterium, which are considered important probiotics associated with the inhibition of pathogenic bacteria and modulation of systemic immune responses [22, 75]. However, in the present study, the abundances of Actinobacteria and Bifidobacterium were greater in APP/PS1 mice than their WT controls at 2 months. This inconsistency might reflect the dynamic and complex nature of microbiota composition under different genetic and environmental circumstances; alternatively, it might indicate that gut microbiota undergoes regulation or compensation in response to early-life inflammatory insult. The brain-gut axis allows bidirectional communication between the CNS and the enteric nervous system, linking the emotional and cognitive centers of the brain with peripheral intestinal functions [76]. Thus, microbiological therapies might be able to restore cognitive health and treat neurological diseases via the modulation of gut microbiota communities.

All animals used in this study were male. During the progression of $\mathrm{AD}$, the male and female mice differ in many aspects, such as body fluid biomarkers, hippocampal atrophy, cognitive decline, and other pathological footprints [77]. Furthermore, the pathological progression in female mice might be affected by the hormone cycle [78]. Previous studies also show that there are sex differences in the gut microbiome [79] and that the gut microbiome can be affected by hormones in female mice [80]. Recent reports show that antibiotic manipulation of the gut microbiome alleviates $\mathrm{AD}$-related pathology in male mice but not female mice $[27,37]$. Thus, to minimize the variation introduced by sex differences, we only included male animals in this study. In humans, the prevalence of $\mathrm{AD}$ or other dementias is $11 \%$ in males and $16 \%$ in females at the age of 71 and older, respectively [81]. This discrepancy might be due to the longer life- span of women or related health factors as well as interactions between genetic factors and sex hormones [82-84]. However, the causes of the observed discrepancy remain unclear.

In summary, the present longitudinal study shows that microbiota composition in WT mice and an $\mathrm{AD}$ mouse model begins diverging early, prior to amyloid plaque formation and neuroinflammation in the cerebral cortex. Meanwhile, at later ages, the microbial composition in $\mathrm{AD}$ mouse models shifts towards an inflammation-related bacterial profile (i.e., increases in the abundance of Escherichia-Shigella and Desulfovibrio), coinciding with abundant microglial accumulation at sites of amyloid deposition in the brain. Nevertheless, the molecular mechanisms and signaling cascades that link the dysregulation of gut microbiota and pathogenesis of $\mathrm{AD}$ await further investigation.

\section{Data Availability}

The data used to support the findings of this study are included within the article, and the Mice Fecal 16S rDNA Amplification Raw Sequence Reads data have been uploaded on the NCBI SRA database: https://www.ncbi.nlm.nih.gov/ bioproject/543965 (project ID: 543965).

\section{Conflicts of Interest}

The authors have no conflicts of interest to report.

\section{Authors' Contributions}

Yijing Chen and Lihua Fang contributed equally to this work.

\section{Acknowledgments}

This study was supported in part by the National Key R\&D Program of China (2018YFE0203600), the National Natural Science Foundation of China (31671047), the Guangdong Provincial Key S\&T Program (2018B030336001), the Guangdong Provincial Fund for Basic and Applied Basic Research (2019B1515130004 and 19201910260000033), the Shenzhen Knowledge Innovation Program (JCYJ20170413165053031 and JCYJ20170413173717055), the Shenzhen-Hong Kong Institute of Brain Science-Shenzhen Fundamental Research Institutions (NYKFKT2019003), and the Guangdong-Hong Kong-Macao Greater Bay Area Center for Brain Science and Brain-Inspired Intelligence Fund (2019001). We also thank the members of the Ma Laboratory and Chen Laboratory for helpful discussion.

\section{Supplementary Materials}

Table S1. Primer sequences for amplification and sequencing of 16S rRNA genes. Table S2. Significantly different taxa between WT and AD mice at phylum, family and genus level. Figure S1. Length of sequence reads of all samples.Figure S2. (A-E) Heatmaps showing the differentially abundant taxa at the OTU level in wild-type (WT) and APP/PS1 mice at 1, 2, 3 , 6, and 9 months of age, respectively. Each column in the heatmap represents a mouse fecal sample, and each row 
represents an OTU-level taxon that is differentially abundant in WT or APP/PS1 mice. The $16 \mathrm{~S}$ rRNA sequence-based cluster tree for the differential OTUs is presented on the left side of the heatmap. $1 \mathrm{~m}, 2 \mathrm{~m}, 3 \mathrm{~m}, 6 \mathrm{~m}, 9 \mathrm{~m}$ indicate mice at $1,2,3,6$, and 9 months of age, respectively.Figure S3. Differentially represented bacterial taxa of WT (A) and APP/PS1 (B) mice at different ages, as revealed by LEfSe analysis. $1 \mathrm{~m}$, $2 \mathrm{~m}, 3 \mathrm{~m}, 6 \mathrm{~m}, 9 \mathrm{~m}$ indicate mice at $1,2,3,6$, and 9 months of age, respectively. (Supplementary Materials)

\section{References}

[1] C. Reitz, C. Brayne, and R. Mayeux, "Epidemiology of Alzheimer disease," Nature Reviews Neurology, vol. 7, no. 3, pp. 137-152, 2011.

[2] A. Cattaneo, N. Cattane, S. Galluzzi et al., "Association of brain amyloidosis with pro-inflammatory gut bacterial taxa and peripheral inflammation markers in cognitively impaired elderly," Neurobiology of Aging, vol. 49, pp. 60-68, 2017.

[3] D. C. Emery, D. K. Shoemark, T. E. Batstone et al., "16S rRNA next generation sequencing analysis shows bacteria in Alzheimer's post-mortem brain," Frontiers in Aging Neuroscience, vol. 9, p. 195, 2017.

[4] D. K. V. Kumar, S. H. Choi, K. J. Washicosky et al., "Amyloid$\beta$ peptide protects against microbial infection in mouse and worm models of Alzheimer's disease," Science Translational Medicine, vol. 8, no. 340, article 340ra72, 2016.

[5] K. Honda and D. R. Littman, "The microbiota in adaptive immune homeostasis and disease," Nature, vol. 535, no. 7610, pp. 75-84, 2016.

[6] M. G. Rooks and W. S. Garrett, "Gut microbiota, metabolites and host immunity," Nature Reviews Immunology, vol. 16, no. 6, pp. 341-352, 2016.

[7] G. De Palma, P. Blennerhassett, J. Lu et al., "Microbiota and host determinants of behavioural phenotype in maternally separated mice," Nature Communications, vol. 6, no. 1, 2015.

[8] E. Y. Hsiao, S. W. McBride, S. Hsien et al., "Microbiota modulate behavioral and physiological abnormalities associated with neurodevelopmental disorders," Cell, vol. 155, no. 7, pp. 14511463, 2013.

[9] P. Zheng, B. Zeng, C. Zhou et al., "Gut microbiome remodeling induces depressive-like behaviors through a pathway mediated by the host's metabolism," Molecular Psychiatry, vol. 21, no. 6, pp. 786-796, 2016.

[10] A. Fasano, N. P. Visanji, L. W. Liu, A. E. Lang, and R. F. Pfeiffer, "Gastrointestinal dysfunction in Parkinson's disease," Lancet Neurology, vol. 14, no. 6, pp. 625-639, 2015.

[11] E. E. Frohlich, A. Farzi, R. Mayerhofer et al., "Cognitive impairment by antibiotic-induced gut dysbiosis: analysis of gut microbiota-brain communication," Brain, Behavior, and Immunity, vol. 56, pp. 140-155, 2016.

[12] S. M. Collins, M. Surette, and P. Bercik, "The interplay between the intestinal microbiota and the brain," Nature Reviews Microbiology, vol. 10, no. 11, pp. 735-742, 2012.

[13] P. Forsythe, J. Bienenstock, and W. A. Kunze, "Vagal pathways for microbiome-brain-gut axis communication," Advances in Experimental Medicine and Biology, vol. 817, pp. 115-133, 2014.

[14] T. C. Fung, C. A. Olson, and E. Y. Hsiao, "Interactions between the microbiota, immune and nervous systems in health and disease," Nature Neuroscience, vol. 20, no. 2, pp. 145-155, 2017.

[15] A. N. Suarez, T. M. Hsu, C. M. Liu et al., "Gut vagal sensory signaling regulates hippocampus function through multi- order pathways," Nature Communications, vol. 9, no. 1, p. 2181, 2018.

[16] H. E. Vuong, J. M. Yano, T. C. Fung, and E. Y. Hsiao, "The microbiome and host behavior," Annual Review of Neuroscience, vol. 40, no. 1, pp. 21-49, 2017.

[17] E. A. Mayer, "Gut feelings: the emerging biology of gut-brain communication," Nature Reviews Neuroscience, vol. 12, no. 8, pp. 453-466, 2011.

[18] B. L. Fredrickson, K. M. Grewen, K. A. Coffey et al., "A functional genomic perspective on human well-being," Proceedings of the National Academy of Sciences of the United States of America, vol. 110, no. 33, pp. 13684-13689, 2013.

[19] C. Brouwers, P. M. Mommersteeg, I. Nyklicek et al., "Positive affect dimensions and their association with inflammatory biomarkers in patients with chronic heart failure," Biological Psychology, vol. 92, no. 2, pp. 220-226, 2013.

[20] J. E. Stellar, N. John-Henderson, C. L. Anderson, A. M. Gordon, G. D. McNeil, and D. Keltner, "Positive affect and markers of inflammation: discrete positive emotions predict lower levels of inflammatory cytokines," Emotion, vol. 15, no. 2, pp. 129-133, 2015.

[21] T. Fulop, J. M. Witkowski, K. Bourgade et al., "Can an infection hypothesis explain the beta amyloid hypothesis of Alzheimer's disease?," Frontiers in Aging Neuroscience, vol. 10, p. 224, 2018.

[22] N. M. Vogt, R. L. Kerby, K. A. Dill-McFarland et al., "Gut microbiome alterations in Alzheimer's disease," Scientific Reports, vol. 7, no. 1, p. 13537, 2017.

[23] Z. Q. Zhuang, L. L. Shen, W. W. Li et al., "Gut microbiota is altered in patients with Alzheimer's disease," Journal of Alzheimer's Disease, vol. 63, no. 4, pp. 1337-1346, 2018.

[24] L. Zhang, Y. Wang, X. Xiayu et al., "Altered gut microbiota in a mouse model of Alzheimer's disease," Journal of Alzheimer's Disease, vol. 60, no. 4, pp. 1241-1257, 2017.

[25] L. Shen, L. Liu, and H. F. Ji, “Alzheimer's disease histological and behavioral manifestations in transgenic mice correlate with specific gut microbiome state," Journal of Alzheimer's Disease, vol. 56, no. 1, pp. 385-390, 2017.

[26] C. Bauerl, M. C. Collado, A. Diaz Cuevas, J. Vina, and G. Perez Martinez, "Shifts in gut microbiota composition in an APP/PSS1 transgenic mouse model of Alzheimer's disease during lifespan," Letters in Applied Microbiology, vol. 66, no. 6, pp. 464-471, 2018.

[27] M. R. Minter, C. Zhang, V. Leone et al., "Antibiotic-induced perturbations in gut microbial diversity influences neuroinflammation and amyloidosis in a murine model of Alzheimer's disease," Scientific Reports, vol. 6, no. 1, 2016.

[28] M. R. Chapman, L. S. Robinson, J. S. Pinkner et al., "Role of Escherichia coli curli operons in directing amyloid fiber formation," Science, vol. 295, no. 5556, pp. 851-855, 2002.

[29] S. C. Wu, Z. S. Cao, K. M. Chang, and J. L. Juang, "Intestinal microbial dysbiosis aggravates the progression of Alzheimer's disease in _Drosophila_" Nature Communications, vol. 8, no. 1, p. 24, 2017.

[30] N. A. Bokulich, S. Subramanian, J. J. Faith et al., "Quality-filtering vastly improves diversity estimates from Illumina amplicon sequencing," Nature Methods, vol. 10, no. 1, pp. 57-59, 2013. 
[31] R. C. Edgar, B. J. Haas, J. C. Clemente, C. Quince, and R. Knight, "UCHIME improves sensitivity and speed of chimera detection," Bioinformatics, vol. 27, no. 16, pp. 21942200, 2011.

[32] R. C. Edgar, "UPARSE: highly accurate OTU sequences from microbial amplicon reads," Nature Methods, vol. 10, no. 10, pp. 996-998, 2013.

[33] R. C. Edgar, "Search and clustering orders of magnitude faster than BLAST," Bioinformatics, vol. 26, no. 19, pp. 2460-2461, 2010.

[34] E. A. Grice, H. H. Kong, S. Conlan et al., "Topographical and temporal diversity of the human skin microbiome," Science, vol. 324, no. 5931, pp. 1190-1192, 2009.

[35] X. Z. Ling, M. J. Kong, F. Liu et al., "Molecular analysis of the diversity of vaginal microbiota associated with bacterial vaginosis," BMC Genomics, vol. 11, no. 1, p. 488, 2010.

[36] N. Segata, J. Izard, L. Waldron et al., "Metagenomic biomarker discovery and explanation," Genome Biology, vol. 12, no. 6, p. R60, 2011.

[37] H. B. Dodiya, T. Kuntz, S. M. Shaik et al., "Sex-specific effects of microbiome perturbations on cerebral $\mathrm{A} \beta$ amyloidosis and microglia phenotypes," The Journal of Experimental Medicine, vol. 216, no. 7, pp. 1542-1560, 2019.

[38] T. Harach, N. Marungruang, N. Duthilleul et al., "Reduction of Abeta amyloid pathology in APPPS1 transgenic mice in the absence of gut microbiota," Scientific Reports, vol. 7, no. 1, 2017.

[39] D. S. Davies, J. Ma, T. Jegathees, and C. Goldsbury, "Microglia show altered morphology and reduced arborization in human brain during aging and Alzheimer's disease," Brain Pathology, vol. 27, no. 6, pp. 795-808, 2017.

[40] P. Kundu, E. Blacher, E. Elinav, and S. Pettersson, "Our gut microbiome: the evolving inner self," Cell, vol. 171, no. 7 , pp. 1481-1493, 2017.

[41] T. Odamaki, K. Kato, H. Sugahara et al., “Age-related changes in gut microbiota composition from newborn to centenarian: a cross-sectional study," BMC Microbiology, vol. 16, no. 1, 2016.

[42] M. J. Claesson, I. B. Jeffery, S. Conde et al., "Gut microbiota composition correlates with diet and health in the elderly," Nature, vol. 488, no. 7410, pp. 178-184, 2012.

[43] T. S. B. Schmidt, J. Raes, and P. Bork, "The human gut microbiome: from association to modulation," Cell, vol. 172, no. 6, pp. 1198-1215, 2018.

[44] W. A. Walters, Z. Xu, and R. Knight, "Meta-analyses of human gut microbes associated with obesity and IBD," FEBS Letters, vol. 588, no. 22, pp. 4223-4233, 2014.

[45] P. W. O'Toole and I. B. Jeffery, "Gut microbiota and aging," Science, vol. 350, no. 6265, pp. 1214-1215, 2015.

[46] M. Sochocka, K. Donskow-Lysoniewska, B. S. Diniz, D. Kurpas, E. Brzozowska, and J. Leszek, "The gut microbiome alterations and inflammation-driven pathogenesis of Alzheimer's disease-a critical review," Molecular Neurobiology, vol. 56, no. 3, pp. 1841-1851, 2019.

[47] V. Rothhammer, D. M. Borucki, E. C. Tjon et al., "Microglial control of astrocytes in response to microbial metabolites," Nature, vol. 557, no. 7707, pp. 724-728, 2018.

[48] P. Perez-Pardo, H. B. Dodiya, P. A. Engen et al., "Role of TLR4 in the gut-brain axis in Parkinson's disease: a translational study from men to mice," Gut, vol. 68, no. 5, pp. 829-843, 2019.
[49] A. Houlden, M. Goldrick, D. Brough et al., "Brain injury induces specific changes in the caecal microbiota of mice via altered autonomic activity and mucoprotein production," Brain, Behavior, and Immunity, vol. 57, pp. 10-20, 2016.

[50] N. R. Shin, T. W. Whon, and J. W. Bae, "Proteobacteria: microbial signature of dysbiosis in gut microbiota," Trends in Biotechnology, vol. 33, no. 9, pp. 496-503, 2015.

[51] C.-H. Chen, C.-L. Lin, and C.-H. Kao, "Irritable bowel syndrome is associated with an increased risk of dementia: a nationwide population-based study," PLoS One, vol. 11, no. 1, p. e0144589, 2016.

[52] A. Keshavarzian, S. J. Green, P. A. Engen et al., "Colonic bacterial composition in Parkinson's disease," Movement Disorders, vol. 30, no. 10, pp. 1351-1360, 2015.

[53] D. W. Kang, J. G. Park, Z. E. Ilhan et al., "Reduced incidence of Prevotella and other fermenters in intestinal microflora of autistic children," PLoS One, vol. 8, no. 7, article e68322, 2013.

[54] M. De Angelis, M. Piccolo, L. Vannini et al., "Fecal microbiota and metabolome of children with autism and pervasive developmental disorder not otherwise specified," PLoS One, vol. 8, no. 10, article e76993, 2013.

[55] C. B. Forsyth, K. M. Shannon, J. H. Kordower et al., "Increased intestinal permeability correlates with sigmoid mucosa alphasynuclein staining and endotoxin exposure markers in early Parkinson's disease," PLoS One, vol. 6, no. 12, article e28032, 2011.

[56] F. Scheperjans, V. Aho, P. A. Pereira et al., "Gut microbiota are related to Parkinson's disease and clinical phenotype," Movement Disorders, vol. 30, no. 3, pp. 350-358, 2015.

[57] A. Asti and L. Gioglio, "Can a bacterial endotoxin be a key factor in the kinetics of amyloid fibril formation?," Journal of Alzheimer's Disease, vol. 39, no. 1, pp. 169-179, 2014.

[58] C.-L. N. Small, S. A. Reid-Yu, J. B. McPhee, and B. K. Coombes, "Persistent infection with Crohn's disease-associated adherentinvasive Escherichia coli_leads to chronic inflammation and intestinal fibrosis," Nature Communications, vol. 4, no. 1, 2013.

[59] D. M. Soares, M. J. Figueiredo, J. M. Martins et al., "A crucial role for IL-6 in the CNS of rats during fever induced by the injection of live E. coli," Medical Microbiology and Immunology, vol. 201, no. 1, pp. 47-60, 2012.

[60] S. J. Soscia, J. E. Kirby, K. J. Washicosky et al., "The Alzheimer's disease-associated amyloid beta-protein is an antimicrobial peptide," PLoS One, vol. 5, no. 3, article e9505, 2010.

[61] B. Routy, E. Le Chatelier, L. Derosa et al., "Gut microbiome influences efficacy of PD-1-based immunotherapy against epithelial tumors," Science, vol. 359, no. 6371, pp. 91-97, 2018.

[62] C. W. Png, S. K. Linden, K. S. Gilshenan et al., "Mucolytic bacteria with increased prevalence in IBD mucosa augment in vitro utilization of mucin by other bacteria," The American Journal of Gastroenterology, vol. 105, no. 11, pp. 2420-2428, 2010.

[63] A. Everard, C. Belzer, L. Geurts et al., "Cross-talk between Akkermansia muciniphila and intestinal epithelium controls diet-induced obesity," Proceedings of the National Academy of Sciences of the United States of America, vol. 110, no. 22, pp. 9066-9071, 2013.

[64] R. Araos, N. Andreatos, J. Ugalde, S. Mitchell, E. Mylonakis, and E. M. C. D'Agata, "Fecal microbiome among nursing home residents with advanced dementia and Clostridium difficile," Digestive Diseases and Sciences, vol. 63, no. 6, pp. 15251531, 2018. 
[65] T. L. Weir, D. K. Manter, A. M. Sheflin, B. A. Barnett, A. L. Heuberger, and E. P. Ryan, "Stool microbiome and metabolome differences between colorectal cancer patients and healthy adults," PLoS One, vol. 8, no. 8, article e70803, 2013.

[66] S. Jangi, R. Gandhi, L. M. Cox et al., "Alterations of the human gut microbiome in multiple sclerosis," Nature Communications, vol. 7, no. 1, 2016.

[67] D. Erny, A. L. Hrabe de Angelis, D. Jaitin et al., "Host microbiota constantly control maturation and function of microglia in the CNS," Nature Neuroscience, vol. 18, no. 7, pp. 965-977, 2015 .

[68] P. M. Smith, M. R. Howitt, N. Panikov et al., "The microbial metabolites, short-chain fatty acids, regulate colonic Treg cell homeostasis," Science, vol. 341, no. 6145, pp. 569-573, 2013.

[69] H. J. Flint, E. A. Bayer, M. T. Rincon, R. Lamed, and B. A. White, "Polysaccharide utilization by gut bacteria: potential for new insights from genomic analysis," Nature Reviews Microbiology, vol. 6, no. 2, pp. 121-131, 2008.

[70] J. Y. Park, J. Choi, Y. Lee et al., "Metagenome analysis of bodily microbiota in a mouse model of Alzheimer disease using bacteria-derived membrane vesicles in blood," Exp Neurobiol, vol. 26, no. 6, pp. 369-379, 2017.

[71] J. Chen, N. Chia, K. R. Kalari et al., "Multiple sclerosis patients have a distinct gut microbiota compared to healthy controls," Scientific Reports, vol. 6, no. 1, 2016.

[72] J. R. Emge, K. Huynh, E. N. Miller et al., "Modulation of the microbiota-gut-brain axis by probiotics in a murine model of inflammatory bowel disease," American Journal of Physiology Gastrointestinal and Liver Physiology, vol. 310, no. 11, pp. G989-G998, 2016.

[73] L. Steenbergen, R. Sellaro, S. van Hemert, J. A. Bosch, and L. S. Colzato, "A randomized controlled trial to test the effect of multispecies probiotics on cognitive reactivity to sad mood," Brain, Behavior, and Immunity, vol. 48, pp. 258-264, 2015.

[74] C. D'Mello, N. Ronaghan, R. Zaheer et al., "Probiotics improve inflammation-associated sickness behavior by altering communication between the peripheral immune system and the brain," The Journal of Neuroscience, vol. 35, no. 30, pp. 10821-10830, 2015.

[75] E. Akbari, Z. Asemi, R. Daneshvar Kakhaki et al., "Effect of probiotic supplementation on cognitive function and metabolic status in Alzheimer's disease: a randomized, doubleblind and controlled trial," Frontiers in Aging Neuroscience, vol. 8, 2016.

[76] D. M. Saulnier, Y. Ringel, M. B. Heyman et al., "The intestinal microbiome, probiotics and prebiotics in neurogastroenterology," Gut Microbes, vol. 4, no. 1, pp. 17-27, 2013.

[77] M. T. Ferretti, For the Women's Brain Project and the Alzheimer Precision Medicine Initiative, M. F. Iulita et al., "Sex differences in Alzheimer disease-the gateway to precision medicine," Nature Reviews Neurology, vol. 14, no. 8, pp. 457469, 2018.

[78] R. M. Shansky, "Are hormones a "female problem" for animal research?," Science, vol. 364, no. 6443, pp. 825-826, 2019.

[79] J. G. Markle, D. N. Frank, S. Mortin-Toth et al., "Sex differences in the gut microbiome drive hormone-dependent regulation of autoimmunity," Science, vol. 339, no. 6123, pp. 1084-1088, 2013.

[80] K. Kaliannan, R. C. Robertson, K. Murphy et al., "Estrogenmediated gut microbiome alterations influence sexual dimor- phism in metabolic syndrome in mice," Microbiome, vol. 6, no. 1, p. 205, 2018.

[81] “2020 Alzheimer's disease facts and figures," Alzheimer's \& Dementia, vol. 16, no. 3, pp. 391-460, 2020.

[82] M. Arnold, K. Nho, A. Kueider-Paisley et al., "Sex and _APOE_ $\varepsilon 4$ genotype modify the Alzheimer's disease serum metabolome," Nature Communications, vol. 11, no. 1, p. 1148, 2020.

[83] K. R. Laws, K. Irvine, and T. M. Gale, "Sex differences in cognitive impairment in Alzheimer's disease," World J Psychiatry, vol. 6, no. 1, pp. 54-65, 2016.

[84] A. Paganini-Hill and V. W. Henderson, "Estrogen deficiency and risk of Alzheimer's disease in women," American Journal of Epidemiology, vol. 140, no. 3, pp. 256-261, 1994. 